Phys. Lett. A, 372/17, (2008), 3064-3070. 


\title{
Wave scattering by many small particles embedded in a medium.
}

\author{
A. G. Ramm \\ (Mathematics Department, Kansas State University, \\ Manhattan, KS66506, USA \\ and TU Darmstadt, Germany) \\ ramm@math.ksu.edu
}

\begin{abstract}
Theory of wave scattering by many small bodies is developed under various assumptions concerning the ratio $\frac{a}{d}$, where $a$ is the characteristic dimension of a small body and $d$ is the distance between neighboring bodies $d=O\left(a^{\kappa_{1}}\right), 0<\kappa_{1}<1$. On the boundary $S_{m}$ of every small body an impedance-type condition is assumed $\frac{\partial u_{M}}{\partial N}=\zeta_{m} u_{M}$ on $S_{m}, 1 \leq m \leq M, \zeta_{m}=h_{m} a^{-\kappa}, 0<\kappa, h_{m}$ are constants independent of $a$. The behavior of the field in the region in which $M=M(a) \gg 1$ small particles are embedded is studied as $a \rightarrow 0$ and $M(a) \rightarrow \infty$. Formulas for the refraction coefficient of the limiting medium are derived under the assumptions: a) $\kappa_{1}=(2-\kappa) / 3,0<\kappa \leq 1$, and b) $\kappa_{1}=1 / 3, \kappa>1$. A method for creating materials with a desired refraction coefficient is proposed and justified theoretically on the basis of the above results.
\end{abstract}

PACS: 43.20. + g, 62.40. + d: 78.20. - e.

MSC: 35J05, 35J10, 70F10, 74J25, 81U40, 81V05

KEYWORDS: wave scattering, many-body scattering, condensed matter physics

\section{Introduction}

The theory of wave scattering by small bodies was originated by Rayleigh in 1871 [1]. In [5] (see also [13]) this theory was developed for small bodies 
of arbitrary shapes, analytic formulas for the $S$-matrix for acoustic and electromagnetic (EM) wave scattering by small bodies of arbitrary shapes have been derived. These formulas allow one to calculate the $S$-matrix with any desired accuracy. Analytic formulas for the electric and magnetic polarizability sensors have been derived for bodies of arbitrary shapes [3], [5]. In [4] - [12] a theory of wave scattering by many small bodies embedded in a bounded domain filled by a material with known properties was developed. It was assumed in [10] and [11] that the characteristic size of the small particles (bodies) is $a$, that the distance $d$ between two neighboring particles is is of the order $d=O\left(a^{1 / 3}\right)$, that the total number of the embedded particles $M=O\left(\frac{1}{a}\right)$, and that the boundary condition on the boundary $S_{m}$ of $m$-th particle $D_{m}$ is of impedance type:

$$
\frac{\partial u_{M}}{\partial N}=\zeta_{m} u_{M} \quad \text { on } S_{m}, \quad 1 \leq m \leq M
$$

where $N$ is the unit normal to $S_{m}$ directed out of $D_{m}$, and $\zeta_{m}=\frac{h_{m}}{a}$, where $h_{m}, \operatorname{Im} h_{m} \leq 0$, is a constant independent of $a$.

The waves in the original material are described by the equation

$$
L_{0} u_{0}:=\left[\nabla^{2}+k^{2} n_{0}^{2}(x)\right] u_{0}=0 \quad \text { in } \mathbb{R}^{3},
$$

where

$$
n_{0}^{2}(x)=1 \quad \text { in } D^{\prime}=\mathbb{R}^{3} \backslash D,
$$

$D$ is a bounded domain, and $n_{0}^{2}(x)$ is continuous in $D$ (or piecewisecontinuous with a finite number of discontinuities, which are smooth surfaces), $\operatorname{Im} n_{0}^{2} \geq 0$. The scattering solution to (2) satisfies the radiation condition

$$
\begin{aligned}
u_{0} & =e^{i k \alpha \cdot x}+v_{0} \\
\frac{\partial v_{0}}{\partial r}-i k v_{0} & =o\left(\frac{1}{r}\right), \quad r:=|x| \rightarrow \infty .
\end{aligned}
$$

If small particles are embedded in $D$, then the scattering problem consists of finding the solution to the following problem:

$$
\begin{aligned}
L_{0} u_{M} & =0 \quad \text { in } \mathbb{R}^{3} \backslash \bigcup_{m=1}^{M} D_{m}, \\
\frac{\partial u_{M}}{\partial N} & =\zeta_{m} u_{M} \quad \text { on } S_{m}, \quad 1 \leq m \leq M, \\
u_{M} & =u_{0}+v_{M},
\end{aligned}
$$


where $u_{0}$ solves problem $(2),(4),(5)$ and $v_{M}$ satisfies the radiation condition similar to (5).

It is proved in [11] that problem (6) - (8) has a unique solution and this solution is of the form

$$
u_{M}=u_{0}(x)+\sum_{m=1}^{M} \int_{S_{m}} G(x, t) \sigma_{m}(t) d t,
$$

where $G(x, y)$ is the Green function of the operator $L_{0}$ for $M=0$, i.e., in the absence of small particles:

$$
L_{0} G(x, y)=-\delta(x-y) \quad \text { in } \mathbb{R}^{3},
$$

$G$ satisfies the radiation condition (5), and $\sigma_{m}$ solves the equation

$$
u_{e_{N}}-\zeta_{m} u_{e}+\frac{A_{m} \sigma_{m}-\sigma_{m}}{2}-\zeta_{m} T_{m} \sigma_{m}=0 \quad \text { on } S_{m}
$$

Here $u_{e}$ is the effective field acting on the $m$-th particle:

$$
\begin{gathered}
u_{e}(x):=u_{e}(x, a):=u_{e}^{(m)}(x):=u_{M}(x)-\int_{S_{m}} G(x, t) \sigma_{m}(t) d t, \quad x \in \mathbb{R}^{3}, \\
A_{m} \sigma_{m}:=2 \int_{S_{m}} \frac{\partial G(s, t)}{\partial N_{s}} \sigma_{m}(t) d t, \quad T_{m} \sigma_{m}:=\int_{S_{m}} G(s, t) \sigma_{m}(t) d t .
\end{gathered}
$$

It was proved in [11] that

$$
G(x, y)=\frac{1}{4 \pi|x-y|}[1+O(|x-y|)], \quad|x-y| \rightarrow 0,
$$

and one can differentiate formula (14).

The following result is also proved in [11]. Assume that $D_{m}$ is a ball of radius $a$ centered at a point $x_{m}$. Let $h(x)$ be an arbitrary continuous function in $D, \operatorname{Im} h(x) \leq 0, \Delta_{p} \subseteq D$ be any subdomain of $D$, and $\mathcal{N}\left(\Delta_{p}\right)$ be the number of particles in $\Delta_{p}$. Assume that

$$
\mathcal{N}\left(\Delta_{p}\right)=\frac{1}{a} \int_{\Delta_{p}} N(x) d x[1+o(1)], \quad a \rightarrow 0,
$$

where $N(x) \geq 0$ is a given continuous function in $D$. Let

$$
p(x):=\frac{4 \pi N(x) h(x)}{1+h(x)} .
$$

Finally, assume that $\zeta_{m}:=\frac{h\left(x_{m}\right)}{a}$. Now the result can be formulated: 
Theorem 1 ([11]). Under the above assumptions there exists the limit

$$
\lim _{a \rightarrow 0}\left\|u_{e}(x)-u(x)\right\|_{C(D)}=0 .
$$

The function $u(x)$ solves the problem

$$
\begin{aligned}
L u & :=\left[\nabla^{2}+k^{2}-q(x)\right] u=0 \quad \text { in } \mathbb{R}^{3}, \\
u & =u_{0}+v,
\end{aligned}
$$

where $u_{0}$ satisfies equations (2), (4), (5), the function $v$ satisfies the radiation condition similar to (5), and

$$
q(x):=q_{0}(x)+p(x), \quad n^{2}(x):=1-k^{-2} q(x),
$$

where $p(x)$ is defined in (16),

$$
q_{0}(x):=k^{2}-k^{2} n_{0}^{2}(x),
$$

and $n_{0}^{2}(x)$ is the coefficient in (2).

The aim of this paper is to investigate the behavior of $u_{e}(x)$ when the assumptions $\zeta_{m}=\frac{h\left(x_{m}\right)}{a}, d=O\left(a^{1 / 3}\right), M=O\left(\frac{1}{a}\right)$ are replaced by the following more general assumptions:

$$
\zeta_{m}=\frac{h\left(x_{m}\right)}{a^{\kappa}}, \quad d=O\left(a^{\kappa_{1}}\right), \quad M=O\left(\frac{1}{a^{3 \kappa_{1}}}\right),
$$

where $\kappa>-1$ and $0 \leq \kappa_{1}<1$ are parameters.

If $\kappa_{1}=1$, then the distance between neighboring particles is of the order of the size of a small particle. This is a special case which is not covered by a rigorous theory. If $\kappa_{1}$ is close to 1 , then the distance between neighboring particles is very close to the order $a$ of the size of a small particle. This is of interest in applications.

In [11] the theory was developed in detail in the case $\kappa=1, \kappa_{1}=\frac{1}{3}$.

The questions we are interested in this paper are: 
1) For what ranges of $\kappa$ and $\kappa_{1}$ the limit $u(x)$ of $u_{e}(x)$, as $a \rightarrow 0$, does exist?

2) What is the equation which this limit $u(x)$ solves?

The answers we give are:

1) If $-1<\kappa<1$ and $\kappa_{1}=\frac{2-\kappa}{3}$, then the limit (17) exists,

$\sigma_{m}=-\frac{h\left(x_{m}\right) u_{e}\left(x_{m}\right)}{a^{\kappa}}(1+o(1)), \quad Q_{m}=-4 \pi h\left(x_{m}\right) a^{2-\kappa} u_{e}\left(x_{m}\right)(1+o(1))$,

and the limiting function $u(x)$ solves the following equation:

$$
u(x)=u_{0}(x)-4 \pi \int_{D} G(x, y) h(y) N(y) u(y) d y .
$$

Therefore, $u$ solves equation (18) with $q(x)$ given by $(20), q_{0}(x)$ given by (21), and

$$
p(x)=4 \pi h(x) N(x),
$$

where $N(x) \geq 0$ is defined by the formula:

$$
\mathcal{N}\left(\Delta_{p}\right)=\frac{1}{a^{3 \kappa_{1}}} \int_{\Delta_{p}} N(x) d x[1+o(1)], \quad a \rightarrow 0,
$$

for any open subset $\Delta_{p} \subset D$ of $D$.

2) If $\kappa>1$ then, as $a \rightarrow 0$,

$$
Q_{m}=-4 \pi u_{e}\left(x_{m}\right) a(1+o(1)), \quad \sigma_{m}=-\frac{u_{e}\left(x_{m}\right)}{a}(1+o(1)),
$$

and the limit (17) exists if $\kappa_{1}=\frac{1}{3}$. The limiting function $u(x)$ solves equation (23) with $h(y)=1$ and $N(x)$ defined by (25).

The function $u(x)$ also solves equation (18) with $q(x)$ given by $(20), q_{0}(x)$ given by (21), and $p(x)$ given by (24) with $h(x)=1$.

In both cases, $0<\kappa<1$ and $\kappa>1$, we have $\kappa_{1}<2 / 3$. This implies that the total volume of the embedded particles tends to zero as $a \rightarrow 0$.

Indeed, the order of the total number of the embedded particles is $O\left(a^{-3 \kappa_{1}}\right)$, and the total volume of the embedded particles is of the order $O\left(a^{3-3 \kappa_{1}}\right) \rightarrow 0$ as $a \rightarrow 0$. 
Let us make a remark about the case when $\kappa_{1}=\frac{2-\kappa}{3}=1$. In this case the distance between neighboring particles is of the order of the size $a$ of the particle in the scale of powers of $a$. If $\kappa_{1}=1$, then $\kappa=-1$ and $\zeta_{m}=h\left(x_{m}\right) a$. Moreover, one has:

$$
Q_{m} \sim a^{3}\left[\frac{4 \pi}{3} \Delta u_{e}\left(x_{m}\right)-4 \pi h\left(x_{m}\right) u_{e}\left(x_{m}\right)\right], \quad a \rightarrow 0,
$$

where $\Delta=\nabla^{2}$ is the Laplacean, and

$$
\sigma_{m} \sim u_{e_{N}}-\operatorname{hau}_{e}\left(x_{m}\right), \quad a \rightarrow 0 .
$$

The quantity

$$
I_{m}:=\left|G\left(x, x_{m}\right) Q_{m}\right|=O\left(a^{3-\kappa_{1}}\right), \quad a \rightarrow 0,
$$

and

$$
J_{m}=\left|\int_{S_{m}}\left[G(x, t)-G\left(x, x_{m}\right)\right] \sigma_{m}(t) d t\right|=O\left(a^{2-2 \kappa_{1}} a^{2}\right), \quad a \rightarrow 0 .
$$

The relation $J_{m} \ll I_{m}$ holds as $a \rightarrow 0$ provided that the relation

$$
a^{4-2 \kappa_{1}} \ll a^{3-\kappa_{1}}
$$

holds. This relation holds if $\kappa_{1}<1$.

The relation $J_{m} \ll I_{m}$ allows us to use formula (35), see below, i.e., approximate the exact formula (31) by an approximate formula (35) with an error which tends to zero as $a \rightarrow 0$.

Assuming $\kappa_{1}=1$ and $I_{m} \gg J_{m}$, one gets

$$
u_{e}(x)=u_{0}+\sum_{m=1}^{M} G\left(x, y^{(p)}\right)\left[\frac{4 \pi}{3} \Delta u_{e}\left(y^{(p)}\right)-4 \pi h\left(y^{(p)}\right) u_{e}\left(y^{(p)}\right)\right] a^{3} \mathcal{N}\left(\Delta_{p}\right) .
$$

From the definition of $\mathcal{N}\left(\Delta_{p}\right)$ one gets:

$$
a^{3} \mathcal{N}\left(\Delta_{p}\right)=\frac{a^{3}}{a^{3 \kappa_{1}}} \int_{\Delta_{p}} N(x) d x[1+o(1)] \approx a^{3-3 \kappa_{1}} N\left(y^{(p)}\right)\left|\Delta_{p}\right|,
$$

where $o(1)$ tends to zero as $a \rightarrow 0$. For the limit of the sum in formula (26) to exist as $a \rightarrow 0$, it is necessary and sufficient that $3=3 \kappa_{1}$, i.e., $\kappa_{1}=1$. 
If $\kappa_{1}=1$, then the limit of $u_{e}(x)$, as $a \rightarrow 0$ and $\max _{p}$ diam $\Delta_{p} \rightarrow 0$, is the function $u(x)$, which solves the equation

$$
u(x)=u_{0}(x)+\int_{D} G(x, y)\left[\frac{4 \pi}{3} \Delta u(y)-4 \pi h(y) u(y)\right] N(y) d y .
$$

Applying operator $L_{0}=\nabla^{2}+k^{2}-q_{0}(x)$ to (28) and using equation (10), one gets

$$
L_{0} u=-\left[\frac{4 \pi}{3} \Delta u-4 \pi h(x) u(x)\right] N(x) .
$$

Thus

$$
\left[1+\frac{4 \pi}{3} N(x)\right] \nabla^{2} u+k^{2} u-q_{0}(x) u-4 \pi h(x) N(x) u(x)=0 .
$$

The solution $u(x)$ to equations (18) or (30) is a locally $H_{l o c}^{2}$ function, where $H_{l o c}^{2}$ is the Sobolev space of twice differentiable in $L^{2}$-sense functions on every bounded open subset of $\mathbb{R}^{3}$. This local smoothness, namely the inclusion $u \in H_{l o c}^{2}\left(\mathbb{R}^{3}\right)$, follows from known results on elliptic regularity, provided that the coefficients $q_{0}(x)$ and $h(x) N(x)$ are in $L_{l o c}^{2}$. If these coefficients are smoother, then $u$ is smoother.

The assumption $\kappa_{1}<1$ allows us to prove that formula (35) of Section 2 is a good approximation of $u$ as $a \rightarrow 0$.

The equations for the effective field in the medium, derived under the assumption $\kappa_{1}=1$, are approximate. They are the more accurate the smaller is the parameter $\Gamma:=a / d$, where $d=\Gamma^{-1} a$ is the distance between neighboring particles, and the parameter $\Gamma$ here is assumed independent of $a$.

In Section 2 we prove the results listed in the answers given above to the two basic questions.

In Section 3 a recipe is given for creating materials with the desired refraction coefficient.

In Section 4 conclusions are stated.

In [2] a theory of wave scattering by randomly distributed isotropic point scatterers is considered under the assumption that the "scattering forces " of individual point scatterers are known. Our theory has virtually no intersection with Foldy's theory: we do not assume the scatterers to be isotropic point scatterers, we do not assume the "scattering forces" to be known, we do not assume random distribution of scatterers, on the contrary, the distribution of the scatterers is defined by formula (25), in which the continuous 
non-negative function $N(x)$ is chosen as we wish, and, finally, the impedance boundary conditions (7) play a crucial role in our theory, while in Foldy's theory there is no surface of the point scatterers, and, therefore, no boundary conditions.

\section{Proofs}

In the proofs we use some arguments from [11]. Let $D_{m}:=\left\{x:\left|x-x_{m}\right|<a\right\}$.

Case 1). Consider first the case $\kappa<1$. Let us write the exact formula (9) as follows:

$$
u_{M}(x)=u_{0}(x)+\sum_{m=1}^{M} G\left(x, x_{m}\right) Q_{m}+\sum_{m=1}^{M} \int_{S_{m}}\left[G(x, t)-G\left(x, x_{m}\right] \sigma_{m}(t) d t\right.
$$

where $x_{m}$ is the center of the ball $D_{m}$ and

$$
Q_{m}:=\int_{S_{m}} \sigma_{m}(t) d t
$$

One has the following estimates (see [11]):

$$
|G(x, y)| \leq \frac{c}{|x-y|}, \quad|\nabla G(x, y)| \leq c \max \left(\frac{k}{|x-y|}, \frac{1}{|x-y|^{2}}\right)
$$

where $c>0$ stands for various constants independent of $a$.

Let us estimate $Q_{m}$. Eventually we want to derive sufficient condition for the relation

$$
I_{m}:=\left|G\left(x, x_{m}\right) Q_{m}\right| \gg\left|\int_{S_{m}}\left[G(x, t)-G\left(x, x_{m}\right)\right] \sigma_{m}(t) d t\right|:=J_{m}
$$

to hold as $a \rightarrow 0$ and $\left|x-x_{m}\right| \gg a$. This relation allows one to rewrite the exact formula (31) as an approximate formula:

$$
u_{M}=u_{0}(x)+\sum_{m=0}^{M} G\left(x, x_{m}\right) Q_{m}, \quad\left|x-x_{m}\right| \gg a,
$$

the error of which tends to zero as $a \rightarrow 0$. 
To derive a formula for $Q_{m}$, integrate (11) over $S_{m}$ and use the divergence theorem to get:

$$
\frac{4}{3} \pi a^{3} \Delta u_{e}\left(x_{m}\right)-\frac{h\left(x_{m}\right)}{a^{\kappa}} u_{e}\left(x_{m}\right) 4 \pi a^{2}=Q_{m}+\frac{h\left(x_{m}\right)}{a^{\kappa}} \int_{S_{m}} d x \int_{S_{m}} \frac{\sigma_{m}(t)}{4 \pi|s-t|} d t .
$$

One has

$$
\int_{S_{m}} d s \int_{S_{m}} \frac{\sigma_{m}(t) d t}{4 \pi|s-t|}=\int_{S_{m}} d t \sigma_{m}(t) \int_{S_{m}} \frac{d s}{4 \pi|s-t|}=a Q_{m} .
$$

Here we have used the formula

$$
\int_{S_{m}:=\left\{s:\left|s-x_{m}\right|=a\right\}} \frac{d s}{4 \pi|s-t|}=a, \quad t \in S_{m} .
$$

Thus, (36) yields:

$$
Q_{m}=\frac{\frac{4}{3} \pi a^{3} \Delta u_{e}\left(x_{m}\right)-4 \pi h\left(x_{m}\right) u_{e}\left(x_{m}\right) a^{2-\kappa}}{1+h\left(x_{m}\right) a^{1-\kappa}} .
$$

If $\kappa<1$ and $a \rightarrow 0$, then (39) implies

$$
Q_{m}=-4 \pi h\left(x_{m}\right) u_{e}\left(x_{m}\right) a^{2-\kappa}[1+o(1)], \quad a \rightarrow 0 .
$$

This is the formula for $Q_{m}$ which we wanted to derive.

If $a \ll 1$, then a formula for $\sigma_{m}$ can be derived as follows. The function $u_{e}$ does not change at a small distance of order $a$. Therefore one can assume that in a neighborhood of $D_{m}$ the function $u_{e}$ is a constant, and one considers a static problem of finding $\sigma_{m}$ :

$$
u_{M}(x)=u_{e}\left(x_{m}\right)+\int_{S_{m}} \frac{\sigma_{m}(t) d t}{4 \pi|x-t|}, \quad \frac{\partial u_{M}}{\partial N}=\zeta_{m} u_{M} \text { on } S_{m} .
$$

We look for the solution $\sigma_{m}=c a^{\gamma}$, where $c$ and $\gamma$ are constants. In this case we have:

$$
\int_{S_{m}} \frac{\sigma_{m}(t) d t}{4 \pi|x-t|}=c a^{\gamma} \frac{a^{2}}{\left|x-x_{m}\right|}, \quad\left|x-x_{m}\right|=O(a) \geq a .
$$

Using the impedance boundary condition (41), neglecting $\frac{\partial u_{e}}{\partial N}$, and choosing the origin at the point $x_{m}$, one gets

$$
-c a^{\gamma} \frac{a^{2}}{a^{2}}=\frac{h\left(x_{m}\right)}{a^{\kappa}}\left[u_{e}\left(x_{m}\right)+c a^{\gamma} \frac{a^{2}}{a}\right] .
$$


From the above equation one derives:

$$
c a^{\gamma}=-\frac{h\left(x_{m}\right) u_{e}\left(x_{m}\right)}{a^{\kappa}\left[1+h\left(x_{m}\right) a^{1-\kappa}\right]} .
$$

If $0<\kappa<1$ and $a \rightarrow 0$, then equation (43) implies $\gamma=-\kappa$ and $c=$ $-h\left(x_{m}\right) u_{e}\left(x_{m}\right)$, so

$$
\sigma_{m}=-\frac{h\left(x_{m}\right) u_{e}\left(x_{m}\right)}{a^{\kappa}}[1+o(1)], \quad a \rightarrow 0 .
$$

This is the formula for $\sigma_{m}$ which we wanted to derive.

Let us find sufficient conditions for the relation (34) to hold.

Using estimates (33) and (39), one obtains:

$$
G\left(x, x_{m}\right) Q_{m}=O\left(\frac{a^{2-\kappa}}{a^{\kappa_{1}}}\right), \quad a \rightarrow 0, \quad\left|x-x_{m}\right| \geq d=O\left(a^{\kappa_{1}}\right), \quad 0<\kappa<1 .
$$

Using (33) and (44) one gets:

$$
J_{m}=O\left(\frac{a}{a^{2 \kappa_{1}}} a^{2-\kappa}\right)=O\left(a^{3-\kappa-2 \kappa_{1}}\right), \quad a \rightarrow 0,\left|x-x_{m}\right| \geq d .
$$

For (34) to hold it is sufficient to have:

$$
a^{3-\kappa-2 \kappa_{1}} \ll a^{2-\kappa-\kappa_{1}}, \quad a \rightarrow 0 .
$$

This relation holds if $\kappa_{1}<1$.

Thus, let us assume that $\kappa<1$ and $\kappa_{1}<1$, and use formulas (35) and (40) to get

$$
u_{M}(x)=u_{0}(x)-\sum_{m=1}^{M} G\left(x, x_{m}\right) 4 \pi h\left(x_{m}\right) u_{e}\left(x_{m}\right) a^{2-\kappa}, \quad\left|x-x_{m}\right| \geq d .
$$

Now we want to pass to the limit $a \rightarrow 0$ in equation (48). To do this, let us partition the domain $D$ into a union of small cubes $\Delta_{p}$ centered at points $y^{(p)}$ and having no common interior points. The side of $\Delta_{p}$ is $b \gg a$. The number $\mathcal{N}\left(\Delta_{p}\right)$ of small particles in $\Delta_{p}$ by formula (25) is:

$$
\mathcal{N}\left(\Delta_{p}\right)=\frac{1}{a^{3 \kappa_{1}}} \int_{\Delta_{p}} N(x) d x[1+o(1)]=\frac{1}{a^{3 \kappa_{1}}} N\left(y^{(p)}\right)\left|\Delta_{p}\right|[1+o(1)],
$$


where $o(1)$ in the second equation tends to zero as diam $\Delta_{p}$ tends to zero, and $\left|\Delta_{p}\right|$ is the volume of the cube $\Delta_{p}$. Write the sum in equation (48) as

$$
\begin{aligned}
\sum_{p} G\left(x, y^{(p)}\right) 4 \pi h\left(y^{(p)}\right) u_{e}\left(y^{(p)}\right) a^{2-\kappa} \sum_{x_{m} \in \Delta_{p}} 1 \\
=\sum_{p} G\left(x, y^{(p)}\right) 4 \pi h\left(y^{(p)}\right) u_{e}\left(y^{(p)}\right) \frac{a^{2-\kappa}}{a^{3 \kappa_{1}}} N\left(y^{(p)}\right)\left|\Delta_{p}\right|(1+o(1)) .
\end{aligned}
$$

The sum in (50) is a Riemannian sum for the integral

$$
\int_{D} G(x, y) 4 \pi h(y) N(y) u_{e}(y) d y
$$

The limit of the sum in (50), as $a \rightarrow 0$, exists if and only if $2-\kappa=3 \kappa_{1}$, i.e.,

$$
\kappa_{1}=(2-\kappa) / 3
$$

Note that if $0<\kappa<1$, then $1 / 3<\kappa_{1}<2 / 3$.

In the region $\left|x-x_{m}\right| \geq d$ one has:

$$
\left|u_{M}(x)-u_{e}(x)\right| \leq O\left(\frac{a}{a^{\kappa_{1}}}\right)=o(1), \quad a \rightarrow 0 .
$$

The number of small particles in a unit cube is $O\left(\frac{1}{d^{3}}\right)=O\left(\frac{1}{a^{3 \kappa_{1}}}\right)$ if $d=$ $O\left(a^{\kappa_{1}}\right)$, where $d$ is the distance between two neighboring particles.

Let us summarize the result.

Lemma 1. Assume that $f$ and $N$ are arbitrary fixed continuous functions in $D$, where $N \geq 0$ is the function in (25), which defines the number of small particles in any subdomain $\Delta_{p}$ of $D$. Then there exists the following limit:

$$
\lim _{a \rightarrow 0} a^{2-\kappa} \sum_{m=1}^{M} f\left(x_{m}\right)=\int_{D} f(x) N(x) d x,
$$

where $M$ is the total number of points $x_{m}$ in $D$, and $2-\kappa=3 \kappa_{1}$.

We assume that the functions $h(y), u_{e}(y)$ and $G(x, y)$ are continuous functions of $y$, so the error of replacing, for example, $h\left(y_{m}\right)$ by $h\left(y^{(p)}\right)$, where $y_{m} \in \Delta_{p}$, goes to zero as diam $\Delta_{p} \rightarrow 0$.

The function $G(x, y)$ is not continuous as $y \rightarrow x$, but $G(x, y)$ is absolutely integrable, so one may remove a small neighborhood of the singular point $x$ 
in the integral (51) and the change of this integral will be negligible if the neighborhood is sufficiently small.

The function $h$ is at our disposal, and we choose it to be continuous.

The continuity of $u_{e}$ and of its limit $u$ follows from the relation $u \in$ $H_{l o c}^{2}\left(\mathbb{R}^{3}\right)$. A more detailed argument is given in [11].

Assuming that $\kappa_{1}=(2-\kappa) / 3$ and passing to the limit $a \rightarrow 0$ in (48) yields equation (23). Applying to this equation the operator $L_{0}$ and using equations (2) and (10), one gets equation (18) with $q(x)$ given by (20) and $p(x)$ given by $(24)$.

We have proved all the claims in the answer to question 1).

Note that if $\kappa<0$, then the impedance parameter $h a^{-\kappa}$ tends to zero as $a \rightarrow 0$.

Case 2). Let us justify the answer to question 2). We assume now that $\kappa>1$. Then (39) implies

$$
Q_{m}=-4 \pi u_{e}\left(x_{m}\right) a[1+o(1)], \quad a \rightarrow 0,
$$

and equation (43) yields

SO

$$
c a^{\gamma}=-\frac{u_{e}\left(x_{m}\right)}{a}
$$

$$
\gamma=-1, \quad c=-u_{e}\left(x_{m}\right)
$$

and

$$
\sigma_{m}=-\frac{u_{e}\left(x_{m}\right)}{a}[1+o(1)], \quad a \rightarrow 0 .
$$

Let us check when the relation (34) holds, i.e., when formula (35) is valid, in other words, when formula (35) yields an accurate approximation of $u_{M}$, defined by formula (31).

From (33) and (53) we conclude, using the relation $d=O\left(a^{\kappa_{1}}\right)$, that

$$
\left|G\left(x, x_{m}\right) Q_{m}\right|=O\left(\frac{a}{d}\right)=O\left(a^{1-\kappa_{1}}\right), \quad a \rightarrow 0, \quad\left|x-x_{m}\right| \geq d .
$$

Furthermore, using (55) and (33), one gets:

$$
J \leq O\left(\frac{a}{a^{2 \kappa_{1}}} a^{2-1}\right)=O\left(a^{2-2 \kappa_{1}}\right), \quad a \rightarrow 0 .
$$


The relation (34) holds if $a^{2-2 \kappa_{1}} \ll a^{1-\kappa_{1}}$, that is, if $\kappa_{1}<1$.

Let us assume that $\kappa_{1}<1$, so that formula (35) is applicable. We repeat the arguments given below formula (48). Due to formula (53), formula (48) now takes the form:

$$
u_{M}(x)=u_{0}(x)-\sum_{m=1}^{M} G\left(x, x_{m}\right) 4 \pi u_{e}\left(x_{m}\right) a, \quad\left|x-x_{m}\right| \geq d .
$$

We conclude from this formula that $u_{e}(x)$ tends to the limit $u(x)$, and $u$ solves the equation:

$$
u(x)=u_{0}(x)-4 \pi \int_{D} G(x, y) N(y) u(y) d y
$$

provided that

$$
\kappa_{1}=\frac{1}{3}
$$

and $N(x)$ is defined by the formula (25) for any subdomain $\Delta \subset D$, where $\mathcal{N}(\Delta)$ is the number of small particles in $\Delta$.

Applying the operator $L_{0}$ to (59) one gets equation (18) for $u(x)$, with $q(x)$ given by $(20), q_{0}(x)$ given by $(21)$, and $p(x)$ given by the formula

$$
p(x)=4 \pi N(x) .
$$

Since $N(x) \geq 0$, the function $p(x)$ is nonnegative.

The assumption $\kappa>1$ leads to the equation (18) with the potential $q(x)$ which can vary much less than in the case $\kappa \leq 1$, because the function $h(x)$ does not enter in the definition of $q(x)$ when $\kappa>1$.

\section{A recipe for creating materials with a de- sired refraction coefficient}

If $\kappa \in(0,1)$ and $\kappa_{1}=(2-\kappa) / 3$, then equations (18), (20) and (24) hold. Thus, given $n_{0}^{2}(x)$ and $n^{2}(x)$, one calculates

$$
p(x)=k^{2}\left[n_{0}^{2}(x)-n^{2}(x)\right]:=p_{1}(x)+i p_{2}(x), \quad p_{2}:=\operatorname{Im} p .
$$

Calculating $p(x)$ by formula (62) is Step 1 of our recipe. This is a trivial step. 
From (24) and (62) one gets an equation for finding $h(x):=h_{1}(x)+i h_{2}(x)$, $h_{2}:=\operatorname{Im} h$, and $N(x) \geq 0$ :

$$
4 \pi\left[h_{1}(x)+i h_{2}(x)\right] N(x)=p_{1}(x)+i p_{2}(x) .
$$

Thus

$$
N(x) h_{1}(x)=\frac{p_{1}(x)}{4 \pi}, \quad N(x) h_{2}(x)=\frac{p_{2}(x)}{4 \pi} .
$$

These equations are much easier to solve for $h_{1}(x), h_{2}(x)$ and $N(x)$, than the equations derived in [11].

There are infinitely many solutions $\left\{h_{1}, h_{2}, N\right\}$ of the two equations (64) for the three unknown functions $h_{1}, h_{2}, N(x), h_{2} \leq 0, N \geq 0$. For example, one may take $N(x)$ to be an arbitrary positive constant, and then $h_{1}$ and $h_{2}$ are uniquely determined by equations (64). The condition $\operatorname{Im} n^{2}(x) \geq 0$ implies $\operatorname{Im} p=p_{2} \leq 0$, which agrees with the inequalities $h_{2} \leq 0, N \geq 0$. One takes $N(x)=h_{1}(x)=h_{2}(x)=0$ at the points at which $p_{1}(x)=p_{2}(x)=0$. At the points at which $|p(x)|>0$, one may take

$$
N(x)=N=\text { const }, \quad h_{1}(x)=\frac{p_{1}(x)}{4 \pi N}, \quad h_{2}(x)=\frac{p_{2}(x)}{4 \pi N} .
$$

Finding the functions $h(x)=h_{1}(x)+i h_{2}(x)$ and $N(x) \geq 0$ by formula (65) is Step 2 of our recipe. This is also a trivial step.

Let us describe Step 3 of our recipe. This step is non-trivial, but it is of purely technological nature, it has nothing to do with the theory.

Let us partition $D$ into a union of small cubes $\Delta_{p}$, which have no common interior points, and which are centered at the points $y^{(p)}$, and embed in each cube $\Delta_{p}$ the number

$$
\mathcal{N}\left(\Delta_{p}\right)=\left[\frac{1}{a^{2-\kappa}} \int_{\Delta_{p}} N(x) d x\right]
$$

of small balls $D_{m}$ of radius $a$, centered at the points $x_{m}$, where $[c]$ stands for the integer nearest to $c>0$, and $\kappa \in(0,1)$. Let us put these balls at 
the distances $O\left(a^{\frac{2-\kappa}{3}}\right)$, and prepare the boundary impedances of these balls equal to $\frac{h\left(x_{m}\right)}{a^{k}}$.

Then the resulting material, which is obtained by embedding small particles into $D$ by the above recipe, will have the desired refraction coefficient $n^{2}(x)$ with an error going to zero as $a \rightarrow 0$.

The technological problems in Step 3 of our recipe are:

a) How does one prepare small balls of radius a with the prescribed boundary impedance $\frac{h\left(x_{m}\right)}{a^{k}}$ ?

b) How does one embed these small balls in a given domain $D$, filled with the known material, according to the requirements formulated in Step 3?

Similarly to [12] one can create by the above recipe materials with a desired spatial dispersion, i.e., one can create the refraction coefficient $n^{2}(x, \omega)$ with a desired $\omega$-dependence, where $\omega$ is the frequency. In particular, one can create materials with negative refraction. This is possible because $N(x)$ one can take frequency-independent, while $h(x, \omega)$ can be taken with any desired frequency dependence.

\section{Conclusions}

Wave scattering by many small particles, embedded into a material with a known refraction coefficient, is studied in this paper.

If $a$ is the characteristic size of a spherical small particle $D_{m}, d=O\left(a^{\kappa_{1}}\right)$ is the distance between the neighboring particles, $\zeta_{m}=\frac{h\left(x_{m}\right)}{a^{k}}$ is the boundary impedance, and $x_{m}$ is the center of $D_{m}$, then the equations are derived, as $a \rightarrow 0$, for the effective field in the medium, consisting of many small particles, embedded in a given material (according to the recipe, derived in Section 2), under the following assumptions:

1) $0<\kappa \leq 1, \kappa_{1}=\frac{2-\kappa}{3}$, and (25) holds,

2) $\kappa>1, \kappa_{1}=\frac{1}{3}$, and (25) holds.

The case $\kappa_{1}=1$ and $\kappa=-1$ is also discussed. In this case $d=O(a)$ is of the order of the size of a small particle.

The results are used for formulating a recipe (see Section 3) for creating materials with a desired refraction coefficient. This recipe is similar to the one, given in [12], [10] in the case $\kappa_{1}=1 / 3$ and $\kappa=1$, but the equations (64) for finding $h_{1}, h_{2}$, and $N$ from the known $p_{1}$ and $p_{2}$ are much simpler, than the equations derived in [12]. 
To the author's knowledge, the theory, developed in this paper, is the first rigorous asymptotic theory of wave scattering by many small particles, embedded in an inhomogeneous medium, under the assumptions that allow for the distance $d=O\left(a^{\kappa_{1}}\right)$ between the neighboring particles to be nearly of the same order of smallness as the particles themselves. More precisely, the parameter $\kappa_{1}<1$ is allowed to be arbitrarily close to 1 .

\section{References}

[1] Landau, L., Lifshitz, E.M., Electrodynamics of continuous medium, Pergamon Press, Oxford, 1960.

[2] Foldy, L., The multiple scattering of waves I. General theory of isotropic scattering by randomly distributed scatterers, Phys. Rev. 67 (1945), pp. 107119 .

[3] Ramm, A.G., Electromagnetic wave scattering by small bodies of arbitrary shapes, in the book: "Acoustic, electromagnetic and elastic scattering-Focus on T-matrix approach" Pergamon Press, N. Y. 1980. 537-546. (ed. V. Varadan).

[4] Ramm, A.G., Equations for the self-consistent field in random medium, Phys.Lett. A, 312, N3-4, (2003), 256-261.

[5] Ramm, A.G., Wave Scattering by Small Bodies of Arbitrary Shapes, World Sci. Publ., Singapore, 2005.

[6] Ramm, A.G., Distribution of particles which produces a "smart" material, Jour. Stat. Phys., 127, N5, (2007), 915-934.

[7] Ramm, A.G., Inverse scattering problem with data at fixed energy and fixed incident direction, Nonlinear Analysis: Theory, Methods and Applications, doi:10.1016/j.na.2007.06.047

[8] Ramm, A.G., Wave scattering by small impedance particles in a medium, Phys. Lett A368, N1-2,(2007), 164-172.

[9] Ramm, A.G., Materials with the desired refraction coefficients can be made by embedding small particles, Phys. Lett. A 370, N5-6, (2007), $522-527$. 
[10] Ramm, A.G., Scattering by many small bodies and applications to condensed matter physics, EPL (Europ. Physics Lett.) 80, (2007), 44001.

[11] Ramm, A.G., Many-body wave scattering by small bodies and applications, J. Math. Phys., 48, 10, (2007), 103511.

[12] Ramm, A.G., A recipe for making materials with negative refraction in acoustics, Phys. Lett. A, 372/13, (2008), 2319-2321.

[13] Ramm, A.G., Inverse Problems, Springer, Berlin, 2005. 